\title{
Has the identification of rectal hypersensitivity any implication in the clinical outcome of irritable bowel syndrome?
}

\author{
S. Izquierdo, E. Rey, M. García Alonso, C. Almansa and M. Díaz-Rubio \\ Service of Digestive Diseases. Hospital Clínico San Carlos. Madrid, Spain
}

\begin{abstract}
Background: sixty percent of patients with irritable bowel disease (IBS) are hypersensitive to rectal distension. It is uncertain to what extend the identification of this abnormality has an impact in the clinical outcome.

Objective: to evaluate if rectal hypersensitivity is associated with a different clinical outcome, prognosis and use of medical resources.

Material and methods: patients with IBS (Rome II criteria) who underwent a rectal distension study at least one year before were eligible if they have not been included in any research protocol since then. We reviewed how many times in the last year they came to emergency room, underwent an endoscopy, and consulted a gastroenterologist or other medical physician for any reason. Also, a telephone interview was done by a gastroenterology fellow using a structured questionnaire to evaluate the frequency and severity of their symptoms in the last year and last month.

Results: a total of 52 patients were eligible and 38 were included. Forteen were not included because inability to made a phone contact or did not consent to phone interview. Twenty six patients were hypersensitive and 12 normosensitive. Both groups had similar symptoms (frequency and severity) but hypersensitive patients visited less to the gastroenterologist $(1.2 \pm 0.2$ vs. $2.9 \pm$ 0.6 times yearly, $p<0.01$ ).

Conclusion: identification of rectal hypersensitivity to distension is associated to less consultation to gastroenterologist, although severity and frequency of symptoms are not modified. Finding of an objective explanation of the symptoms seems to help patients to understand their disease, leading to a decrease in resources' use.
\end{abstract}

Key words: Irritable bowel syndrome. Sensitivity. Use of health resources.
Recibido: 20-04-04.

Aceptado: 11-11-04.

Correspondencia: Sonia Izquierdo Rubio. Servicio de Aparato Digestivo. Hospital Clínico San Carlos. C/ Profesor Martín Lagos, s/n. 28040 Madrid. Tel.: 913303 000.e-mail: soniaizq@yahoo.es
Izquierdo S, Rey E, García Alonso M, Almansa C, Díaz-Rubio $M$. Has the identification of rectal hypersensitivity any implication in the clinical outcome of irritable bowel syndrome? Rev Esp Enferm Dig 2005; 97: 223-228.

\section{INTRODUCTION}

Irritable bowel syndrome (IBS) is a functional disorder with a high prevalence, which ranges between $3.3 \%$ (1) and $22 \%$ (2) depending on the population evaluated and diagnostic criteria used. Twelve percent of individuals with IBS consult their primary care physician, and more than $40 \%$ consult their gastroenterologist $(3,4)$, which represents a high use of health resources (medical consultations, hospitalization, and drug prescriptions) and additional costs because of work absenteeism (4,5-7).

A very important pathophysiological mechanism of IBS is visceral hypersensitivity (8). The identification of hypersensitivity may be useful as a diagnostic test $(9,10)$. Also, a positive test may have a specific effect: to provide objective data to facilitate patient understanding of the functional origin of their symptoms, and indirectly improve physician-patient relationship; to our knowledge, this effect has not been evaluated thus far. So, a positive test may reduce health care utilization, reducing primary care and gastroenterology visits (11).

For that reason, the objective of our study is to evaluate if rectal hypersensitivity is associated with a different clinical outcome, prognosis, and use of sanitary resources in patients with IBS.

\section{MATERIAL AND METHODS}

Patients: Patients meeting the Rome II criteria for irritable bowel syndrome who underwent a rectal distention 
test at least one year before this study were eligible. The rectal distention test was performed according to a technique and criteria previously published $(10,12,13)$. Only patients that had been followed by one gastroenterologist (ER) and had never participated in diagnostic or therapeutic protocols were included. At the time of the rectal distention test, all patients had frequent (more than $50 \%$ of days) and severe symptoms (interference with daily activities), which had been unsuccessfully treated with at least 2 courses of usual drugs -fiber supplements, antispasmodic agents, laxatives, etc.- for 8 weeks. According to the results of the rectal distention test, patients were classified as normosensitive or hypersensitive.

A phone interview with a structured questionnaire was performed to all patients enrolled by 2 persons unaware of the results of the rectal distention test. The structured questionnaire incorporated clinical items such as frequency and severity of symptoms, and also drugs used during the last month and year. The severity of symptoms was evaluated in a scale ranging from 1 to 4 , according to the influence of these symptoms in quality of life (1: "I can perform all my usual activities"; 2: "I can perform all my usual activities, but I cannot forget that I have pain"; 3: "I can perform my activities but I have to introduce some changes in my way of life"; 4: "I cannot perform my usual activities").

In addition, we reviewed a database of Specialized Care at Madrid's $7^{\text {th }}$ Health Area, to which all patients belonged. This is a computerized database including all health services provided to patients by the public system in the $7^{\text {th }}$ Health Area. For each patient, the times during the last year they had presented to the emergency room, underwent an endoscopy or radiographic procedure, and visited a gastroenterologist or other specialist were recorded. Data were expressed as the number of events per year. During the phone interview we verified directly with patients that they had not looked for health services out of the public system.

A descriptive and analytical analysis was made with the collected data using the statistical program SPSS, version 10. Quantitative variables were expressed as mean \pm SEM, and qualitative variables as percentage. Comparisons between quantitative variables were made using an analysis of variance (ANOVA), and qualitative ones using the Chi square test. Alpha level was set at 0.05 .

\section{RESULTS}

A total of 52 patients were eligible but 14 were not included because of inability to make phone contact or because they did not consent to the phone interview, so finally 38 patients were included. Of these 38 patients included, 26 were hypersensitive to rectal distention, whereas 12 were normosensitive. Table I shows the basal features of both groups, with no significant differences between them.
Table I. Basal characteristics of patients

\begin{tabular}{lccc}
\hline & Hypersensitive & Normosensitive & $p$ \\
\hline Number of patients & 26 & 12 & \\
Age (years) & $48.9 \pm 2.9$ & $54.9 \pm 5.0$ & NS \\
Female: male & $21: 7$ & $9: 3$ & NS \\
Time between test and interview (years) & $2.29 \pm 0.16$ & $2.59 \pm 0.27$ & NS \\
Clinical subgroups & & & \\
$\quad$ Alternating-IBS & 12 & 1 & \\
$\quad$ Diarrhea-IBS & 9 & 3 & $p<0.05$ \\
$\quad$ Constipation-IBS & 5 & 8 & \\
Discomfort threshold (mmHg) & $19.08 \pm 0.95$ & $36 \pm 2.15$ & $p<0.001$ \\
Maximal tolerable distention (mmHg) & $27,23 \pm 1,70$ & $39.33 \pm 1.69$ & $p<0.001$ \\
\hline
\end{tabular}

\section{Outcome of symptoms}

Almost all patients remained symptomatic at the time of the phone interview, as shown in table II; there were no significant differences between normosensivite and hypersensitive patients.

Table II. Clinical features at follow-up

\begin{tabular}{lccc}
\hline & Hypersensitive & Normosensitive & $p$ \\
\hline Patients with symptoms last year (\%) & $91.6 \%$ & $91.6 \%$ & NS \\
Months with symptoms during last year & $5.8 \pm 0.8$ & $8.1 \pm 1.3$ & NS \\
Patients with symptoms last month (\%) & $79.1 \%$ & $75 \%$ & NS \\
Days with symptoms during last month & $15.8 \pm 2.2$ & $23 \pm 3.6$ & NS \\
Severity of symptoms (1-4) & $2.2 \pm 0.8$ & $1.9 \pm 0.3$ & NS
\end{tabular}

\section{Use of health resources}

As shown in table III, hypersensitive patients used less health resources than normosensitive patients, although significant differences were only found regarding gastroenterology consultations $(1.2 \pm 0.2$ vs. $2.9 \pm 0.6$ times yearly, $\mathrm{p}<0.01)$.

Table III. Use of resources (events per patient per year)

\begin{tabular}{lccc}
\hline & Hypersensitive & Normosensitive & $p$ \\
\hline Gastroenterology visits (no./ year) & $1.2 \pm 0.2$ & $2.9 \pm 0.6$ & $<0.01$ \\
Other specialties' visits (no./ year) & $4.4 \pm 1.1$ & $6.4 \pm 1.5$ & $\mathrm{NS}$ \\
Visits to emergency departments (no./ year) & $0.3 \pm 0.1$ & $0.3 \pm 0.1$ & $\mathrm{NS}$ \\
Hospitalizations (no./ year) & $0.05 \pm 0.02$ & $0.1 \pm 0.07$ & $\mathrm{NS}$ \\
Endoscopies (no./ year) & $0.03 \pm 0.02$ & $0.05 \pm 0.05$ & $\mathrm{NS}$ \\
Radiographic studies (no./ year) & $0.61 \pm 0.21$ & $0.9 \pm 0.3$ & $\mathrm{NS}$ \\
\hline
\end{tabular}

\section{DISCUSSION}

IBS is a frequent functional bowel disorder $(1,2)$ and one of the most common reasons for patient referral to a gastroenterologist $(3,4)$. IBS is associated with increased 
healthcare resources use and costs; also, patients with IBS have less quality of life than patients without it (3$5,15)$. Medical treatment aims to reduce symptoms and to increase quality of life in patients with IBS, but sometimes this is not possible, especially in those with moderate to severe IBS.

Illnycky et al. (11) demonstrated recently that direct contact with an specialist provides an educational intervention that is associated with fewer visits to specialists and a decrease in sanitary costs. In 1995, Owens et al. (6) explored the therapeutic value of physician-patient interaction; their results suggest that a strong physician-patient interaction was critical to reduce the number of return visits for IBS-related symptoms.

In our study we show that understanding symptoms' mechanisms may have a positive impact on the clinical outcome of patients with IBS. How could a sensitivity test help to explain the nature of symptoms? Without a sensitivity test, we explain symptoms as motility-related ("spasm" or "vague bowel") or sensitivity-related ("feeling normal stimuli more"). Irrespective of the doctor's ability to do it, and the patient's capacity to understand it, acceptance of the functional origin of symptoms is not always achieved. It is easier to explain hypersensitivity to a patient when he or she is actually experiencing it. Thus, the main difference in managing these groups of patients is the explanation provided. We only could explain symptoms to normosensitive patients in the conventional way mentioned before, but hypersensitive patients received an explanation that was sustained in an unquestionable and easily understandable fact (15).

To our knowledge, no study had previously evaluated visceral hypersensitivity as a potential outcome factor. Our data indicate that the natural evolution of hypersensitive patients is similar to that of normosensitive ones, although the studied population represents a subgroup of patients with moderate-severe IBS symptoms. Other factors, including psychological factors, may also be important in the development and evolution of symptoms $(16,17)$.

Our study shows that hypersensitive patients visited fewer physicians. To this day no study has identified the capacity of a diagnostic test to simultaneously decrease consultation rates without changing IBS symptoms. Multiple factors influence physician consultations by patients with IBS (18).

Our study does not allow any particular reason to be established, but the most reasonable explanation is that the identification of an objective mechanism for symptoms impacts on the learned behavior of chronic disease, thereby eliminating uncertainty regarding digestive symptoms. So, hypersensitive patients would not feel the need to visit physicians when with symptoms, and would just learn "to better coexist with them".

In summary, our study shows that the identification of visceral hypersensitivity entails a diminution of gastroenterology consultations in the absence of symptom improvement.

\section{REFERENCES}

1. Mearin F, Badia X, Balboa A, et al. Irritable bowel syndrome prevalence varies enormously depending on the employed diagnostic criteria: comparison of Rome II vs. previous criteria in a general population. Scand J Gastroenterol 2001; 36: 1155-61.

2. Jones R, Lydeard S. Irritable bowel syndrome in the general population. BMJ 1992; 304: 87-90.

3. Camilleri M, Williams DE. Economic burden of irritable bowel syndrome proposed strategies to control expeditures. Pharmaoeconomics 2000; 17: 331-8.

4. Talley NJ, Gabriel SE, Harmsen WS, et al. Medical costs in community individuals with irritable bowel syndrome. Gastroenterology 1995; 109: 1736-41.

5. Badia X, Mearin F, Balboa A, Baró E, Caldwell E, Cucala M, et al. Burden of illness in irritable bowel syndrome comparing Rome I and Rome II criteria. Pharmacoeconomics 2002; 20: 749-58.

6. Hahn BA, Yan S, Strassels S. Impact of irritable bowel syndrome on quality of life and resource use in the United States and United Kingdom. Digestion 1999; 60: 77-81.

7. Longstreth GF, Wilson A, Knight K, Wong J, Chiou CF, Barghout V. Irritable bowel syndrome, health care use, and costs: A U.S. managed care perspective. Am J Gastroenterol 2003; 98: 600-7.

8. Mertz H. Review article: visceral hipersensitivity. Aliment Pharmacol Ther 2003; 17: 623-33.

9. Bouin M, Plourde V, Biivin M, Riberdy M, Lupien F, Laganiere M, et al. Rectal distention testing in patients with irritable bowel syndrome: sensitivity, specificity, and predictive values of pain sensory thresholds. Gastroenterology 2002; 122: 1771-7.

10. Rey E, Díaz-Rubio M. Alteraciones de la sensibilidad visceral en el síndrome del intestino irritable. Med Clin Monogr (Barc) 2003; 4: 246.

11. Ilnyckyj A, Graff LA, Blanchard JF, Bernstein CN. Therapeutic value of a gastroenterology consultation in irritable bowel syndrome. Aliment Pharmacol Ther 2003; 17: 871-80.

12. Rey E, Díaz-Rubio M. Prevalencia de hipersensibilidad rectal en el síndrome de intestino irritable y sus subgrupos clínicos. Rev Esp Enferm Dig 2002; 94: 247-52.

13. Rey E. ¿Cuál es el mejor protocolo de distensión para estudiar la sensibilidad rectal en el síndrome de intestino irritable? Rev Esp Enferm Dig 2002; 94: 211-5.

14. Leong SA, Barghout V, Birnbaum HG, Thibeault CE, Ben-Hamadi $\mathrm{R}$, Frech F, et al. The economic consequences of irritable bowel syndrome. Arch Intern Med 2003; 163: 929-35.

15. Owens DM, Nelson DK, Talley NJ. The irritable bowel syndrome: long-term prognosis and the physician-patient interaction. Ann Intern Med 1995; 122: 107-12.

16. Sykes MA, Blanchard EB, Lackner J, Keefer L, Krasner S. Psychopathology in irritable bowel syndrome: support for a psychophysiological model. J Behav Med 2003; 26: 361-72.

17. Locke GR 3rd, Weaver AL, Melton LT 3rd, Talley NJ. Psycosocial factors are linked to functional gastrointestinal disorders: a population based nested case-control study. Am J Gastroenterol 2004; 99: 350-7.

18. Koloski NA, Talley NJ, Boyce PM. Predictors of health care seeking for irritable bowel syndrome and nonulcer dyspepsia: a critical review of the literature on symptoms and psychosocial factors. Am J Gastroenterol 2001; 96: 1340-9. 